\title{
HPV E7-mediated NCAPH ectopic expression regulates the carcinogenesis of cervical carcinoma via PISK/AKT/SGK pathway
}

\author{
Meng Wang ${ }^{1,2}$, Xiaowen Qiao ${ }^{1,3}$, Tamara Cooper ${ }^{4}$, Wei Pan ${ }^{1}$, Liang Liư ${ }^{4}$, John Hayball ${ }^{4}$, Jiaxiang Lin', Xiujie Cui ${ }^{5}$, \\ Yabin Zhou ${ }^{5}$, Shule Zhang ${ }^{1}$, Ying Zou', Ranran Zhang ${ }^{1}$ and Xiao Wang ${ }^{1,3}$
}

\begin{abstract}
Cervical cancer is one of the most common gynecological tumors in the world, and human papillomavirus (HPV) infection is its causative agent. However, the molecular mechanisms involved in the carcinogenesis of cervical cancer still require clarification. Here we found that knockdown of Non-SMC (Structural Maintenance of Chromosomes) condensin I complex subunit H (NCAPH) gene expression significantly inhibited the proliferation, migration, invasion and epithelial-mesenchymal transition (EMT) of cervical cancer cells in vitro, and restrained xenograft tumor formation in vivo. Intriguingly, HPV E7 could form a positive feedback loop with NCAPH. E7 upregulated NCAPH gene expression via E2F1 which initiated NCAPH transcription by binding to its promoter directly. Silencing of NCAPH reduced E7 transcription via promoting the transition of AP-1 heterodimer from c-Fos/c-Jun to Fra-1/c-Jun. Moreover, the E7mediated NCAPH overexpression was involved in the activation of the PI3KAKT/SGK signaling pathway. In vivo, $\mathrm{NCAPH}$ expression in cervical cancer tissues was significantly higher than which in normal cervix and high-grade squamous intraepithelial lesion (HSIL) tissues, and its expression was significantly correlated with tumor size, depth of invasion and lymph node metastasis. Patients with high NCAPH expression had a significantly better survival outcomes than those with low-expression, suggesting that NCAPH-induced cell proliferation might sensitize cancer cells to adjuvant therapy. In conclusion, our results revealed the role of NCAPH in the carcinogenesis of cervical cancer in vitro and in vivo. The interaction between E7 and NCAPH expands the mechanism of HPV induced tumorigenesis and that of host genes regulating HPV E7.
\end{abstract}

\section{Introduction}

Cervical cancer is one of the most common malignant tumors in women all over the world. Every year, 500,000 new cases occur worldwide, and with a mortality rate over $30 \%{ }^{1}$. Human papillomavirus (HPV) infection is the primary cause of cervical cancer ${ }^{2}$. The HPV genome is composed of double-stranded DNA, encoding seven early

\footnotetext{
Correspondence: Xiao Wang (wangxiao08@sdu.edu.cn)

'Department of Pathology, School of Basic Medical Sciences, Shandong University, Jinan, PR China

2Department of Radiation Oncology, Qilu Hospital, Cheeloo College of Medicine, Shandong University, Jinan, PR China

Full list of author information is available at the end of the article

These authors contributed equally: Meng Wang, Xiaowen Qiao

Edited by $\mathrm{G}$. Blandino
}

and two late open reading frames. The oncogenic protein E7, one of the early expression proteins, is the key oncoprotein leading to malignant transformation of cervical cells ${ }^{3}$. It can regulate cell cycle, inhibit cell apoptosis, and promote cell invasion and metastasis ${ }^{3-7}$. However, HPV infection alone cannot induce the transformation of host cells, but initiates the process towards oncogenesis ${ }^{8,9}$. Once HPV infects the cervical epithelium, subsequent molecular changes involving a cascade of signaling pathways ultimately promote the development and progression of cervical cancer ${ }^{10,11}$. At present, the detailed molecular mechanism involved in this process require further elucidation.

\section{(c) The Author(s) 2020}

(c) (i) Open Access This article is licensed under a Creative Commons Attribution 4.0 International License, which permits use, sharing, adaptation, distribution and reproduction c. in any medium or format, as long as you give appropriate credit to the original author(s) and the source, provide a link to the Creative Commons license, and indicate if changes were made. The images or other third party material in this article are included in the article's Creative Commons license, unless indicated otherwise in a credit line to the material. If material is not included in the article's Creative Commons license and your intended use is not permitted by statutory regulation or exceeds the permitted use, you will need to obtain permission directly from the copyright holder. To view a copy of this license, visit http://creativecommons.org/licenses/by/4.0/. 
Human non-SMC (Structural Maintenance of Chromosomes)-condensin I complex subunit H (NCAPH), encoded by the NCAPH gene, also known as BRRN1 (located on chromosome $2 \mathrm{q} 11.2$ ), functions to maintain the stability of condensin protein complexes and ensure the precise separation of sister chromatids during cell mitosis ${ }^{12-14}$. In 2007, Ryu et al. reported for the first time that the expression of NCAPH was significantly increased in advanced malignant melanoma ${ }^{15}$. In recent years, researchers found that the high expression of NCAPH was associated with poor prognosis in patients with non-small cell lung cancer and prostate cancer ${ }^{16,17}$. Downregulation of NCAPH inhibited the proliferation, migration, and invasion of several cancer cells significantly ${ }^{18-20}$. Moreover, NCAPH was involved in the regulation of mature chromosome condensation and DNA damage ${ }^{21}$. These data suggest that NCAPH may be a key carcinogen involved in the development and progression of human malignant tumors. However, up to now, the function of NCAPH in human cancers is largely unknown, and the underlying molecular mechanism has not been reported.

In this study, we found that reduced expression of $\mathrm{NCAPH}$ can inhibit the malignant growth and the epithelial-mesenchymal transition (EMT) process of cervical cancer cells in vitro, and impede the formation of tumors in nude mice. We confirmed the relationship between NCAPH expression and clinicopathological parameters and prognosis of cervical cancer patients. More importantly, we first elucidated the regulatory loop between HPV E7 and NCAPH, and revealed the regulatory role of NCAPH on the PI3K/AKT/SGK pathway in cervical cancer.

\section{Materials and methods Ethics statement}

Human and animal studies were approved by the ethics committee of Medical School of Shandong University. Written informed consent was obtained from all patients before use of the materials.

\section{Patient population}

Paraffin specimens include 165 cases of invasive cervical squamous cell carcinoma (ICSCC), 34 cases of highgrade squamous intraepithelial lesion (HSIL) and 82 cases of normal cervix collected between March 2005 and March 2013. Fresh specimens include 10 cases of normal cervix and 22 cases of cervical cancer collected from July 2016 to July 2017 at the Department of Pathology, Qilu hospital. Hematoxylin and eosin (HE)-stained sections were reviewed by two experienced pathologists. The diagnoses were made according to the World Health Organization Classification of Tumors. Patient information was obtained from patient medical record room at Qilu Hospital. All patients had no history of other tumors, otherwise they would be excluded from observation.

\section{Follow-up}

Patients were followed-up every 3 months until death or March 2018. Data collected included survival time, diseasefree time, and development of metastases. The period between the date of operation and death was recorded as the overall survival (OS) time. The period between the date of surgery and recurrence or death was noted as the disease-free survival (DFS) time. Patients who were alive at the last follow-up were marked as censored observations.

\section{Bioinformatics analysis of genetic changes and gene expression of NCAPH in cancers}

The cBioPortal database (http://www.cbioportal.org/) was used to explore the genetic variation and proteins coexpressed with NCAPH in cervical cancer. The JASPAR database (http://jaspar.genereg.net/) was used to predict the potential transcription factor that binds to the promoter of NCAPH gene. The GEPIA (http://gepia.cancerpku.cn/) and UALCAN database (http://ualcan.path.uab. $\mathrm{edu} /$ ) were used to determine the differentially expressed genes between normal cervix and cervical cancer samples and to unravel NCAPH gene expression between different normal and cancer tissues. Gene set enrichment analysis (GSEA) was applied to identify the gene sets that were related to NCAPH expression. Data were downloaded from The Cancer Genome Atlas (TCGA, https://tcgadata.nci. nih.gov/tcga/) database and the Gene Expression Omnibus (GEO, https://www.ncbi.nlm.nih.gov/geo/) database. $p<0.05$ was considered statistically significant.

\section{Cell lines and culture}

The cervical cancer cell lines HeLa and SiHa were purchased from the American Type Culture Collection (Manassas, VA, USA). The Human retinal pigment epithelial cells (RPE1-pBabe) and HPV16-E7 expressing RPE1 cells (RPE1-16E7) were kindly provided by Prof. Jianxing Chen (the University of Massachusetts Hospital). All the cells were authenticated using STR profile analysis and tested for mycoplasma contamination recently. HeLa and 293T cells were routinely cultured in Dulbecco's modified Eagle's medium, SiHa cells were maintained in RPMI-1640 medium, and both RPE1-pBabe cell lines were cultured in a 1:1 blend of DMEM and Ham's Nutrient Mixture F12 medium (Gibco BRL, Grand Island, NY, USA). All cell lines were cultured at $37^{\circ} \mathrm{C}$ with $10 \%$ fetal bovine serum (Gibco BRL, Grand Island, NY, USA) and $1 \%$ penicillin-streptomycin.

\section{RNA extraction and real time PCR}

Total RNA was extracted from the cells using TRIzol (Ambion, Calsbad, CA, USA) and cDNA synthesized 
using a first strand cDNA synthesis kit (Toyobo, Osaka, Japan). Real-time PCR was performed using an ABI Prism 7000 Sequence Detection System with SYBR Premix Ex Taq (Takara, Otsu, Japan). The primer sequences for amplification are listed in Supplementary Table S1. GAPDH was used as the internal control.

\section{Small-interfering RNA (siRNA) and plasmid transfection}

Cells were seeded in six-well culture plates at a density of $2 \times 10^{5}$ cells per well, starved in serum-free medium without antibiotics for $24 \mathrm{~h}$, and then transfected with siRNA or plasmid mixed with Lipofectamine 2000 (Invitrogen, Calsbad, CA, USA). The sequences of specific siRNA (GenePharma, Shanghai, China) are listed in Supplementary Table S1. The E2F1 and NCAPH overexpression plasmid, pCMV-E2F1 and pLenti6/V5-DEST$\mathrm{NCAPH}$, were purchased from the Addgene repository (Sidney St, Suite 550A, Cambridge, MA), and mock vector was used as a control.

\section{Western blot analysis}

Cells were scraped, pelleted and lysed using CelLyticTM MT Cell Lysis Reagent (Sigma, St. Louis, USA) for total protein extraction. Protein concentration was determined using the BCA reagent kit (Beyotime, Shanghai, China). Western blot analysis was performed as previously described $^{22}$. Membranes were probed with primary antibodies listed in Supplementary Table S2. GAPDH was used as the internal control. The protein bands were visualized using an enhanced chemiluminescence kit (Beyotime, Shanghai, China) according to the manufacturer's instructions.

\section{Cell proliferation assay}

Cell proliferation was assessed using the Cell-Light ${ }^{\mathrm{TM}}$ EdU DNA Cell Proliferation kit (Ribobio, Guangzhou, China) and the Cell-counting kit (CCK-8) (Dojindo, Japan). For the EdU assay, cells were seeded into wells of 96-well plates at a density of $1 \times 10^{4}$ cells per well. After EdU labeling, $100 \mu \mathrm{L}$ of $1 \times$ Apollo reaction cocktail was added and cells were stained with $100 \mu \mathrm{L}$ of Hoechst 33342 and observed under fluorescence microscope (Olympus, Japan). The percentage of EdU positive cells was defined as the proliferation rate. For the CCK- 8 assay, cells were seeded into wells of 96 -well plates at a density of $3 \times 10^{3}$ cells per well and processed according to the manufacturer's instruction.

\section{Colony formation assay}

Colony formation was measured as described previously $^{22}$. In brief, cells were seeded into wells of six-well plates at 500 cells per well after $48 \mathrm{~h}$ of transfection. After further incubation for 10 days, colonies were stained with Giemsa and counted.

\section{Cell migration and invasion assays}

Migration assays were performed using Transwell inserts $(8.0 \mu \mathrm{m}, 24$-well format; Corning, NY, U.S.). For the invasion assay, the inserts were pre-coated with Matrigel matrix (BD Science, Sparks, MD, U.S.). Cell migration and invasion assays were conducted as described previously ${ }^{23}$.

\section{Plasmid construction and Luciferase assay}

The 3'-UTR region of the NCAPH gene was synthesized and inserted into the pGL3-Basic vector to construct the plasmid pGL3-NCAPH (Biosune Biotechnology, Shanghai, China). The pGL3-NCAPH, pCMV-E2F1, pGL3Basic (mock vector), and pCMV (mock vector) plasmids were co-transfected into $293 \mathrm{~T}$ cells pairwise. The LCR regions of HPV16/18 with or without the mutation in AP1 binding sites were synthesized and inserted into the pGL3-Basic vector to construct the plasmids pGL3HPV16/18 wtLCR (wild type) and pGL3- HPV16/18 mutLCR (mutation type) (Biosune Biotechnology, Shanghai, China). Then the plasmids and mock vector were co-transfected into HeLa and $\mathrm{SiHa}$ cells treated with siNCAPH or 293T cells transfected with NCAPH overexpression plasmid separately. Bright-Glo reagent (BrightGlo $^{\mathrm{TM}}$ Luciferase Assay System; Promega, USA) was added $48 \mathrm{~h}$ post-transfection and Firefly luciferase activity was measured. Each experiment was performed in triplicate.

\section{Immunofluorescence staining}

Cells were cultured in a six-well plate and transfected with the specific siRNA targeting NCAPH. They were then transferred to the cover slips and fixed with cold acetone. After blocking with goat serum, cells were incubated with mouse anti-human primary antibody against Vimentin (1:50) (Proteintech, Wuhan, China) overnight at $4{ }^{\circ} \mathrm{C}$. After washing with phosphate-buffered saline, the slides were stained with tetraethyl rhodamine isothiocyanate-labeled goat anti-mouse secondary antibody and counterstained with 4',6-diamidino-2-phenylindole for imaging of the nuclei.

\section{Immunohistochemistry staining}

The immunohistochemistry staining was performed as described previously ${ }^{22}$. The staining results were judged by two pathologists in a single-blinded manner and graded as follows: 0 (no staining); 1 (light yellow); 2 (yellow brown), and 3 (strong brown). The positive proportion of tumor cells was evaluated as follows: $0(<5 \%$ positive cells); 1 (5-25\% positive cells); 2 ( $26-50 \%$ positive cells); 3 (51-75\% positive cells), and 4 ( $>75 \%$ positive cells). The staining index (SI) was calculated as the score of the staining intensity multiplied by the proportion of positive cells. SI value was graded as $0,(-)$; $\leq 4,(+)$; $5-8,(++)$; and $\geq 9,(+++)$. Specimens scored $(-)$ to $(+)$ were 
considered negative, and others were positive. If the staining interpretation differed between the 2 investigators, the data for the slide was discarded.

\section{Nude mice tumor formation assay}

Lentivirus containing pGV248-NCAPH shRNA and pGV248 control vector (carrying GFP protein) was synthesized by Shanghai Genechem Co. Ltd. HeLa and SiHa cells were infected with the virus according to the manufacturers' instructions in the presence of $1 \mu \mathrm{g} / \mathrm{ml}$ puromycin for 6 weeks. The interference efficacy of $\mathrm{NCAPH}$ expression was confirmed by Western Blot analysis. The 4-week-old male NOD/SCID mice were randomized into two groups with comparable body weight. $1 \times 10^{7} \mathrm{HeLa}$ cells stably interfering with NCAPH and control cells were then injected subcutaneously into the left axillary fossa of the mice. Tumor growth was monitored for 4 weeks in a single-blinded manner. Tumor volume was determined with the formula: Volume $=(\text { width })^{2} \times$ length/2. The hematoxylin and eosin (HE) sections of the tumor mass were observed. Non-invasion of the tumor was defined as intact fibrotic capsule and no adjacent stroma invasion. Tumor invasion was recorded as incomplete encapsulation, muscle or vascular invasion.

\section{Statistical analysis}

SPSS 17.0 software (SPSS Inc., Chicago, IL) was used for statistical analysis. The correlation between NCAPH expression and clinicopathologic characteristics were assessed by Chi-square and Spearman correlation test. The difference of NCAPH expression between normal cervix, HSIL, and cervical cancer was evaluated by Chi-square test. Patients' survival was analyzed by the Kaplan-Meier method and compared using the log-rank test. Analysis of cell biological behaviors with siRNA treatment involved Student's $t$ test. Data are expressed as mean \pm SD. $p<0.05$ was considered statistically significant.

\section{Results}

NCAPH is overexpressed in cervical cancer and different solid tumors in vivo

To explore the role of NCAPH in cervical cancer in vivo, we first determined the NCAPH mRNA level in 10 cases of normal cervical tissues and 22 cases of invasive cervical squamous cell carcinoma (ICSCC) tissues by real-time quantitative PCR. The mRNA level of NCAPH in cervical cancer tissues was significantly higher than that in normal cervical tissues $(p<0.05)$ (Fig. 1A). The results of immunohistochemistry staining showed that $\mathrm{NCAPH}$ was expressed mainly in the nucleus but also in the cytoplasm (Fig. 1B). NCAPH was overexpressed in $12.2 \%$ of normal cervical squamous epithelium (10/82), 14.7\% of high-grade squamous intraepithelial lesion (HSIL) (5/34) and $40.6 \%$ of ICSCC (67/165) (Table 1). The expression of NCAPH in cervical cancer was significantly higher than in normal cervix $(p<0.001)$ and HSIL $(p=0.004)$ separately (Table 1 ; Fig. 1C). Although NCAPH over expression in HSIL was more frequently higher than that in normal cervix, the difference was not statistically significant ( $p$ å 0.05 ) (Table 1). The result was further confirmed by the data from GEO database (Supplementary Fig. S1A-C). Moreover, NCAPH was undetectable in 52 cases of normal cervical columnar epithelium and 40 cases of cervical adenocarcinoma (data not shown). Receiver operating characteristic curves demonstrated that NCAPH expression could clearly separate normal cervix and cervical cancer cases, with the area under curve of 0.8826 (Fig. 1D, $p=0.0006$ ).

To further confirm the important role of NCAPH in cancer, we analyzed the expression of NCAPH in various cancers. The results in GEPIA database showed that the expression of NCAPH was significantly higher in esophageal carcinoma, breast invasive adenocarcinoma, head and neck squamous cell carcinoma, and bladder urothelial carcinoma than that in normal tissues (Fig. 1E). Similar results were obtained by analysis of the data from UALCAN database (Supplementary Fig. S1D).

\section{The correlation of NCAPH with the clinicopathological parameters and patients' outcome}

We next investigated the relationship between the expression of NCAPH and the clinicopathological parameters of cervical cancer patients. Results showed that the expression of NCAPH was significantly associated with tumor size $(p=0.032)$, depth of invasion $(p=0.029)$ and lymph node metastasis $(p=0.041)$, but not related to patients' age, FIGO staging, tumor differentiation, or distant metastasis (Table 2), (Fig. 1F) (all $p$ values $>0.05$ ) .

Furthermore, we assessed the correlation of $\mathrm{NCAPH}$ expression with the patients' prognosis using Kaplan-Meier survival analysis. Of 165 patients, 100 patients were successfully followed up. These 100 patients were divided into two groups: NCAPH-positive (38 cases) and NCAPH-negative group (62 cases). The disease-free survival (DFS) rate and overall survival (OS) rate were $80.6 \%$ (average DFS time: 59.9 months, 95\% CI 53.3-66.6 months) and $82.3 \%$ (average OS time: 62.7 months, 95\% CI 57.1-68.2 months) in NCAPHnegative group, respectively. The DFS rate and OS rate were $84.2 \%$ (average DFS time: 86.1 months, 95\% CI 79.1-93.0 months) and $86.8 \%$ (average OS time: 90.0 months, 95\% CI 83.9-96.1 months) in NCAPHpositive group, respectively. The log-rank test revealed that the difference of survival curves between the two groups was statistically significant $(p<0.05)$ (Fig. 1G, H) demonstrating that patients with higher NCAPH expression tend to have better prognosis. 


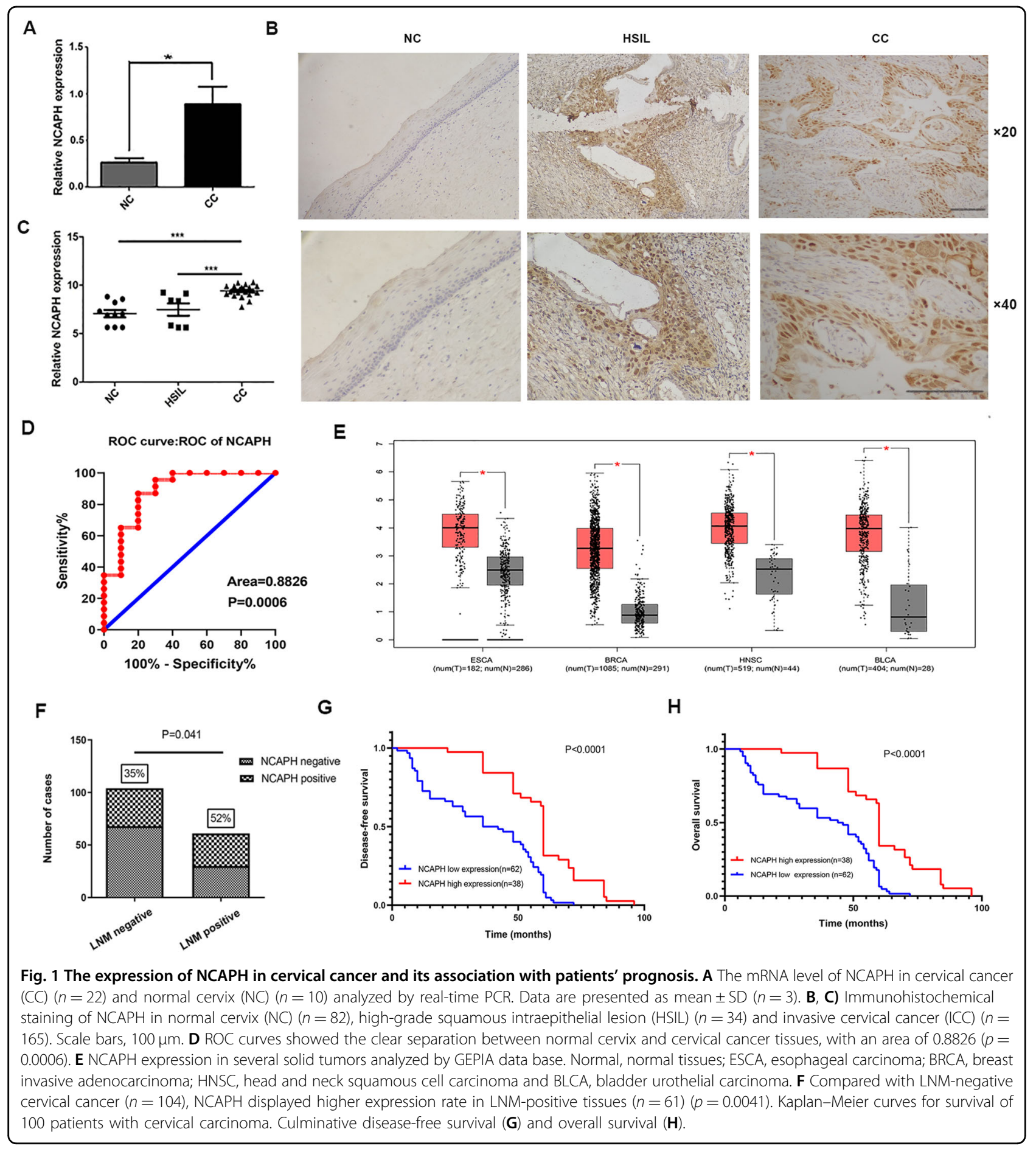

\section{Genetic alterations of NCAPH gene in cervical cancer}

We used cBioPortal to study the genetic changes of $\mathrm{NCAPH}$ gene in all the two cervical cancer studies. In 607 cases of cervical cancer, one case had NCAPH amplification, three cases had missense mutation (Supplementary Fig. S2). The low rate of genetic alteration indicates that the over-expression of NCAPH in cervical cancer is not mainly induced by NCAPH genetic changes.

\section{Reduced NCAPH expression effectively inhibits the proliferation and colony formation of cervical cancer cells}

To evaluate the biological function of NCAPH in cervical cancer, we designed three pairs of specific siRNAs 
Table 1 Expression of NCAPH in cervical tissue samples.

\begin{tabular}{|c|c|c|c|c|c|}
\hline \multirow[b]{2}{*}{ Categories } & \multirow[b]{2}{*}{$n$} & \multicolumn{2}{|l|}{ NCAPH } & \multirow[b]{2}{*}{$P$ value } & \multirow[b]{2}{*}{$x^{2}$} \\
\hline & & Negative & Positive & & \\
\hline normal & 82 & 72 & 10 & 0.714 & 0.135 \\
\hline HSIL & 34 & 29 & 5 & & \\
\hline normal & 82 & 72 & 10 & 0.000 & 20.608 \\
\hline ICSCC & 165 & 98 & 67 & & \\
\hline HSIL & 34 & 29 & 5 & 0.004 & 8.19 \\
\hline ICSCC & 165 & 98 & 67 & & \\
\hline
\end{tabular}

normal normal cervical squamous epithelium, HSIL high-grade squamous intraepithelial lesion, ICSCC invasive cervical squamous cell carcinoma. $p<0.05$ was considered significant (indicated in bold).

Table 2 Clinicopathological characteristics of patients with cervical cancer and NCAPH status.

\begin{tabular}{|c|c|c|c|c|c|}
\hline \multirow[b]{2}{*}{ Categories } & \multirow[b]{2}{*}{$\mathbf{N}$} & \multicolumn{2}{|l|}{ NCAPH } & \multirow[b]{2}{*}{$P$ value } & \multirow[b]{2}{*}{$x^{2}$} \\
\hline & & Negative & Positive & & \\
\hline \multicolumn{6}{|l|}{ Age (y) } \\
\hline$\leq 40$ & 53 & 35 & 18 & 0.232 & 1.429 \\
\hline$>40$ & 112 & 63 & 49 & & \\
\hline \multicolumn{6}{|l|}{ FIGO stage } \\
\hline 1 & 152 & 91 & 61 & 0.671 & 0.180 \\
\hline$\|\&\| \|$ & 13 & 7 & 6 & & \\
\hline \multicolumn{6}{|l|}{ Differentiation grade } \\
\hline Poor & 92 & 51 & 41 & 0.245 & 1.352 \\
\hline Well to moderate & 73 & 47 & 26 & & \\
\hline \multicolumn{6}{|l|}{ Distant metastasis } \\
\hline No & 162 & 96 & 66 & 0.796 & 0.067 \\
\hline Yes & 3 & 2 & 1 & & \\
\hline \multicolumn{6}{|l|}{ Tumor size } \\
\hline$\leq 4 \mathrm{~cm}$ & 132 & 73 & 59 & 0.032 & 4.580 \\
\hline$>4 \mathrm{~cm}$ & 33 & 25 & 8 & & \\
\hline \multicolumn{6}{|l|}{ LN metastasis } \\
\hline No & 104 & 68 & 36 & 0.041 & 4.186 \\
\hline Yes & 61 & 30 & 31 & & \\
\hline \multicolumn{6}{|c|}{ The depth of invasion } \\
\hline$\leq 1 / 2$ & 39 & 29 & 10 & 0.029 & 4.742 \\
\hline$>1 / 2$ & 126 & 69 & 57 & & \\
\hline
\end{tabular}

FIGO International Federation of Gynecology and Obstetrics, LN lymph node. $p<0.05$ was considered significant (indicated in bold).

targeting NCAPH gene, and assessed their interference efficiency in cervical cancer cell lines HeLa and SiHa. Real-time quantitative PCR and Western blot analysis verified that all three siRNAs significantly inhibited the expression of NCAPH in the cells (Fig. 2A-D). NCAPH siRNA \#962 was chosen for subsequent experiments. CCK-8 and Edu assay revealed that when transfected with NCAPH siRNA, the proliferation ability of cervical cancer cells was reduced significantly compared with those transfected with NC siRNA (Fig. 2E-H) (all $p$ values $<$ $0.05)$. Consistent with it, NCAPH was significantly coexpressed with PCNA (a specific marker for proliferation ability) in 308 cases of cervical cancer patients from the cBioportal database (Supplementary Fig. S3). Moreover, the colony formation assay showed that once the expression of $\mathrm{NCAPH}$ was knocked down, the capacity of $\mathrm{HeLa}$ and $\mathrm{SiHa}$ cells to form colonies was significantly decreased compared with those transfected with $\mathrm{NC}$ siRNA (Fig. 2I, J) (all $p$ values $<0.05$ ).

\section{Reduced NCAPH expression significantly inhibits cell migration, invasion, and EMT process in cervical cancer cells}

To determine the effects of NCAPH on the migration and invasion of cervical cancer cells, we performed transwell assays. Results showed that the number of migrating and invasive cells decreased significantly after reducing the expression of NCAPH in HeLa and SiHa cells (Fig. 3A, B; all $p$ values $<0.01$ ). This indicates that the inhibition of $\mathrm{NCAPH}$ expression weakens the migration and invasion capability of HeLa and SiHa cells.

To elucidate whether NCAPH influences the migration and invasion of cancer cells via the regulation of the EMT process, we tested the effects of NCAPH on the expression levels of EMT related proteins in HeLa and SiHa cells by Western Blot analysis and immunofluorescent staining. Results showed that when the cells were transfected with NCAPH siRNA, the protein levels of the mesenchymal markers, such as Vimentin and Snail, decreased significantly compared with those transfected with NC siRNA (Fig. 3C-E). In contrast, the interference of $\mathrm{NCAPH}$ expression significantly increased the expression level of the epithelial marker ZO-1 (Fig. 3C-E). Similarly, the fluorescence signal of Vimentin decreased significantly when the expression of $\mathrm{NCAPH}$ was knocked down in HeLa and SiHa cells (Fig. 3F, G). This suggests that NCAPH promotes the epithelial mesenchymal transition in cervical cancer cells.

\section{Silencing NCAPH expression dramatically impairs the tumor growth, invasion, and EMT process in nude mice}

HeLa cells were infected with lentivirus containing pGV248-NCAPH shRNA and pGV248 control vector separately. Fluorescence microscopy confirmed that $60 \%$ of the tumor cells were successfully infected by the virus (Fig. 4A). Western blot analysis demonstrated that compared with the NC group, NCAPH protein level was dramatically decreased in HeLa cells transfected with pGV248-NCAPH shRNA after 6-weeks of selection using puromycin (Fig. 4B). The selected cells were cultured and injected into the mice for the evaluation of NCAPH function in vivo. Results showed that compared with the 


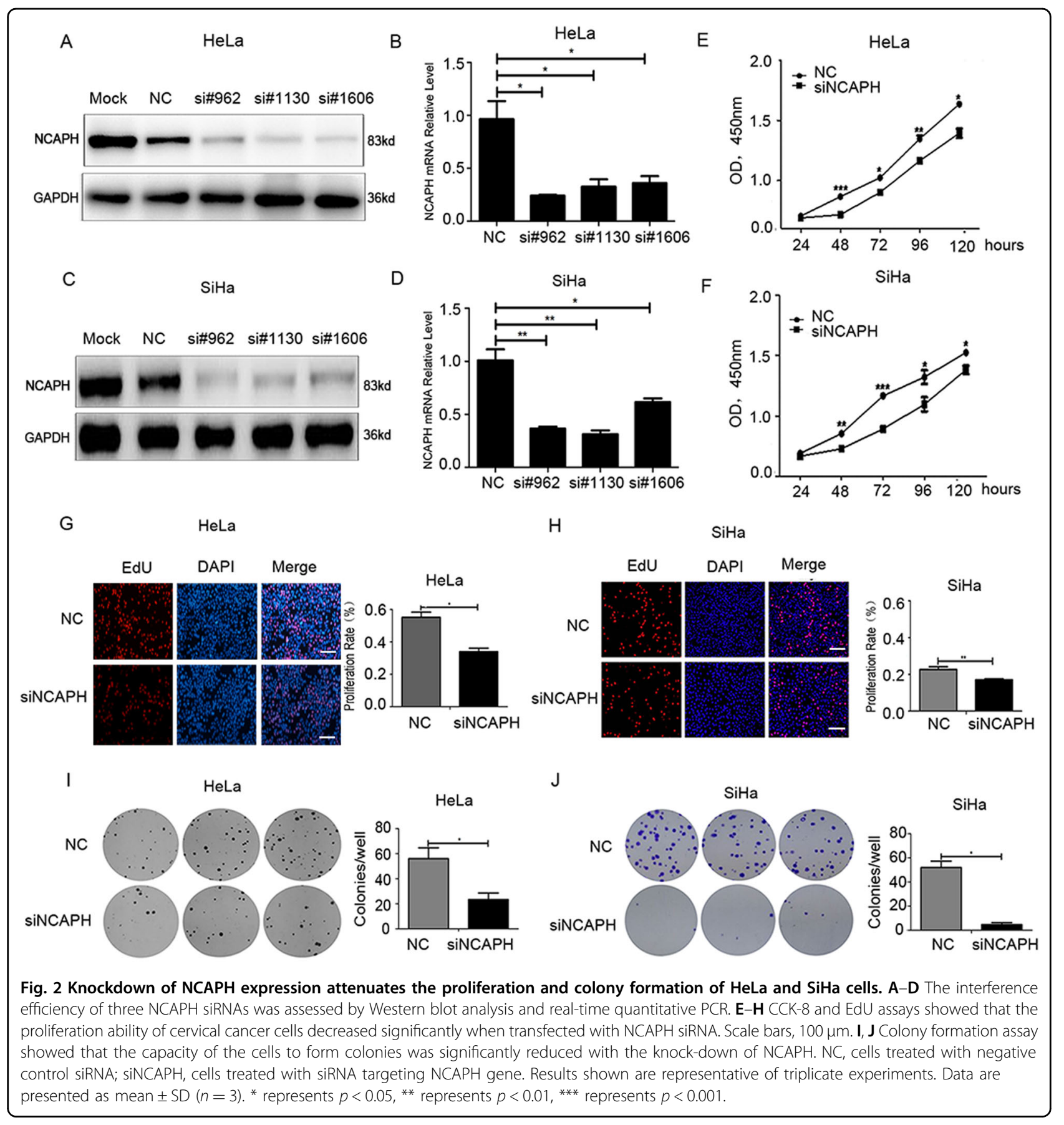

$\mathrm{NC}$ group, the tumor volume was significantly smaller in mice carrying the tumor cells transfected with pGV248NCAPH shRNA (Fig. 4C-E). In addition, tumors derived from control vector exhibited obvious muscle invasion indicating strong invasion ability, while tumors established from pGV248-NCAPH shRNA had an intact fibrotic capsule and less adjacent stroma invasion (Fig. 4F). This indicates that knockdown of NCAPH reduces the tumor formation ability of HeLa cells in vivo.
To further confirm the regulatory role of NCAPH in the EMT process in vivo, we performed immunohistochemistry staining in the mouse graft. The results showed that compared with the control group, the signal of ZO-1 protein was enhanced in mice tumors induced by NCAPH knocking down HeLa cells, while the signal of vimentin was significantly weakened (Fig. 4G) $(p<0.05)$. Thus, both in vitro and in vivo results showed that NCAPH plays a vital role in the regulation of EMT processes in cervical cancer. 


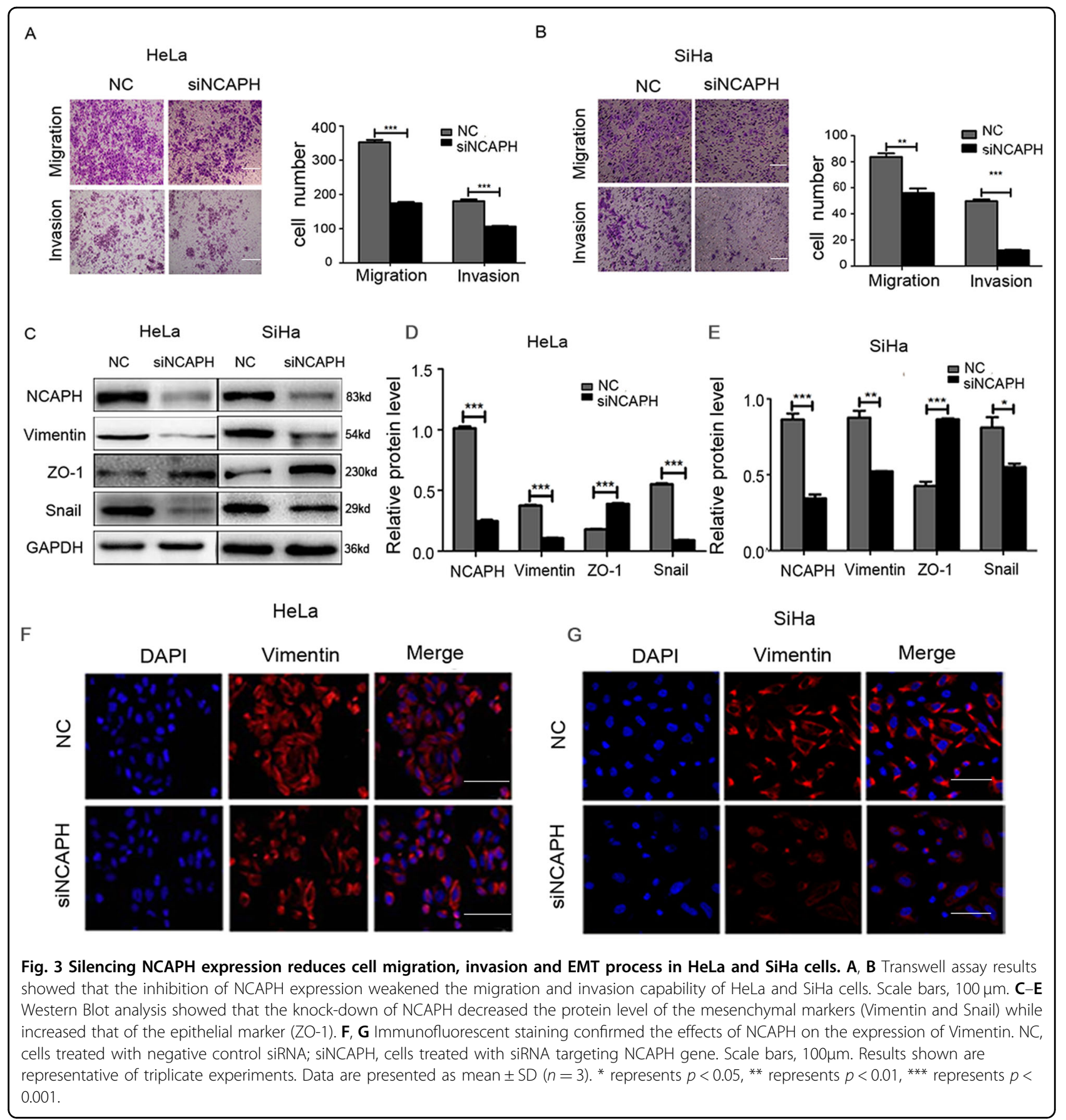

HPV E7 initiates the transcription of NCAPH via the transcription factor E2F1 that binds to the promoter region of NCAPH

By analyzing the data from GEO database (GSE7803), we found that E2F1 expression was significantly increased from normal cervix to cervical cancer and from highgrade intraepithelial lesion (HSIL) to cervical cancer (Fig. 5A). Moreover, GSEA analysis revealed that E2F1 was positively correlated with NCAPH expression (Fig. 5B). JASPAR database showed that E2F1 was a potential transcription factor that binds to the promoter of NCAPH gene (data not shown). To verify the predicted result, we performed the luciferase assay. Results showed that the luciferase activity increased dramatically when the pCMV-E2F1 plasmid was co-transfected with the pGL3$\mathrm{NCAPH}$ plasmid indicating that E2F1 could bind to the promoter region of the NCAPH gene (Fig. 5C) $(p<0.01)$. To verify whether E2F1 could initiate the transcription of NCAPH, we transfected HeLa and SiHa cells with E2F1 siRNA and pCMV-E2F1 plasmid 


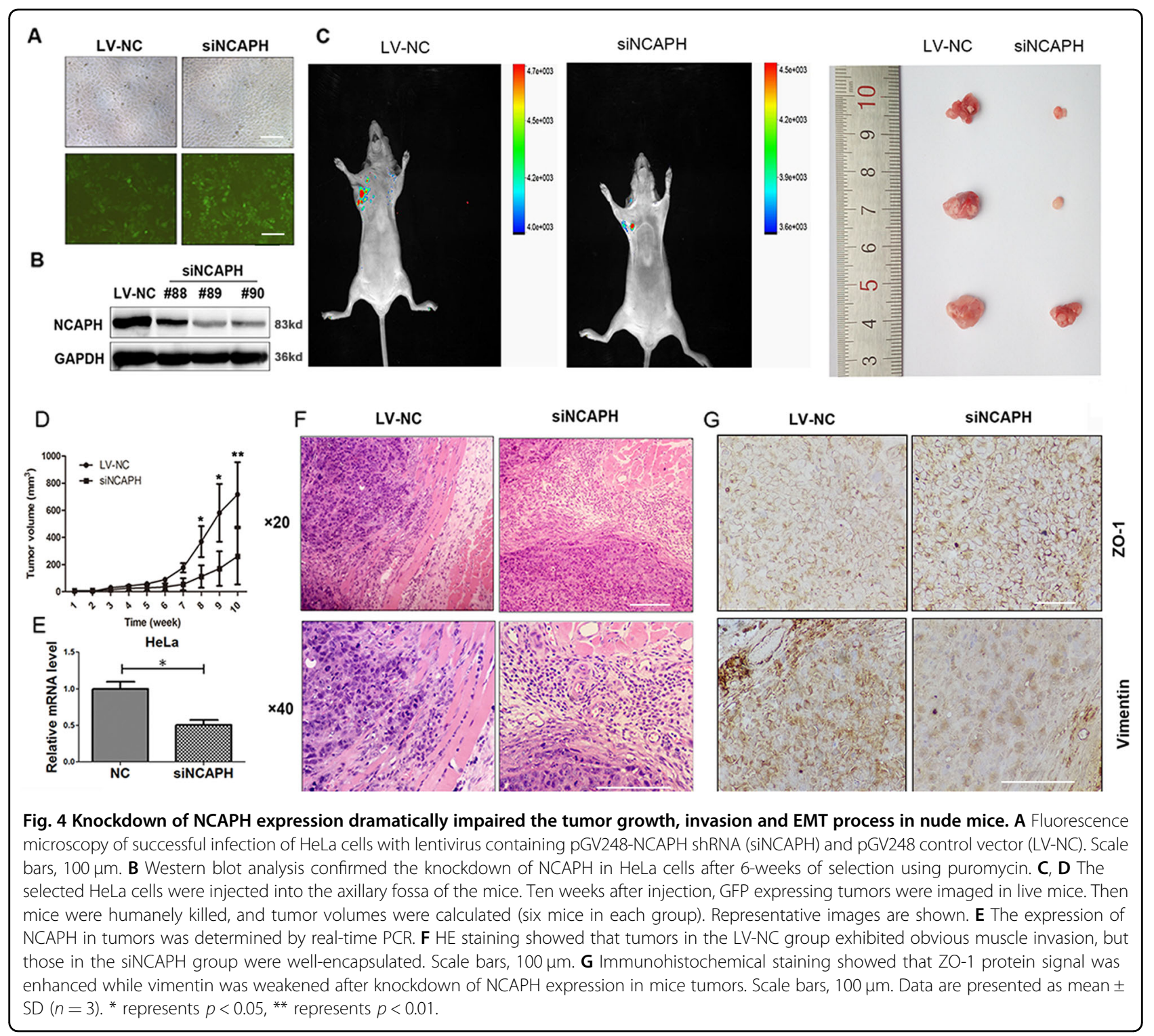

separately. Real-time PCR and Western blot analysis revealed that mRNA and protein levels of NCAPH significantly decreased with the knockdown of E2F1 or increased with the over-expression of E2F1 in cervical cancer cells (Fig. 5D-G) (all the $p$ values $<0.05$ ). Taken together, these results suggested that the transcription factor E2F1 could bind to the promoter region of $\mathrm{NCAPH}$, thereby activating the transcription and subsequent translation of NCAPH gene.

In view of the strong association between HPV E7 and E2F1, we hypothesized that HPV E7 might regulate the expression of NCAPH via E2F1. To test the hypothesis, we transfected specific E7 siRNA into HeLa and $\mathrm{SiHa}$ cells. Results showed that the mRNA and protein levels of E2F1 and NCAPH reduced simultaneously with decreased HPV E7 gene expression (Fig. 5H-J) (all the $p$ values
$<0.05$ ). We next compared the E2F1 and NCAPH expression levels in RPE1-pBabe cells, RPE1-16E7 cells. Western blot analysis demonstrated that the protein levels of E2F1 and NCAPH were significantly higher in RPE116E7 cells compared with RPE1-pBabe cells. Moreover, when RPE1-16E7 cells were transfected with E2F1 siRNA, the elevated NCAPH expression in RPE1-16E7 cells decreased dramatically (Fig. 5K). The data indicated that HPV E7 could up-regulate NCAPH gene expression via E2F1 at the transcriptional level.

\section{Silencing NCAPH downregulates HPV E7 transcription by conversing AP-1 heterodimer from c-Fos/c-Jun to Fra-1/c- Jun}

Intriguingly, when we knocked-down $\mathrm{NCAPH}$ gene expression in $\mathrm{HeLa}$ and $\mathrm{SiHa}$ cells, the mRNA levels of 


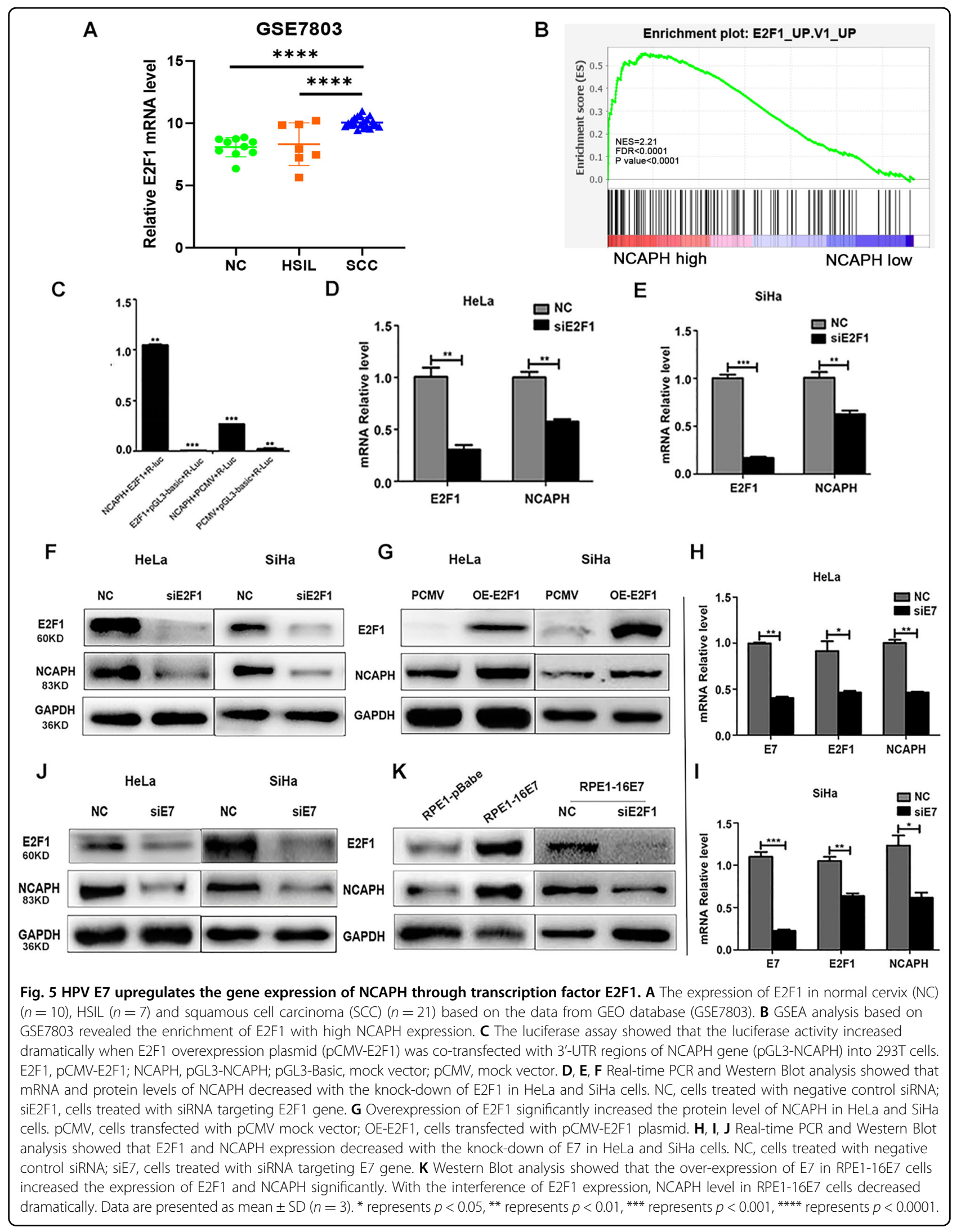




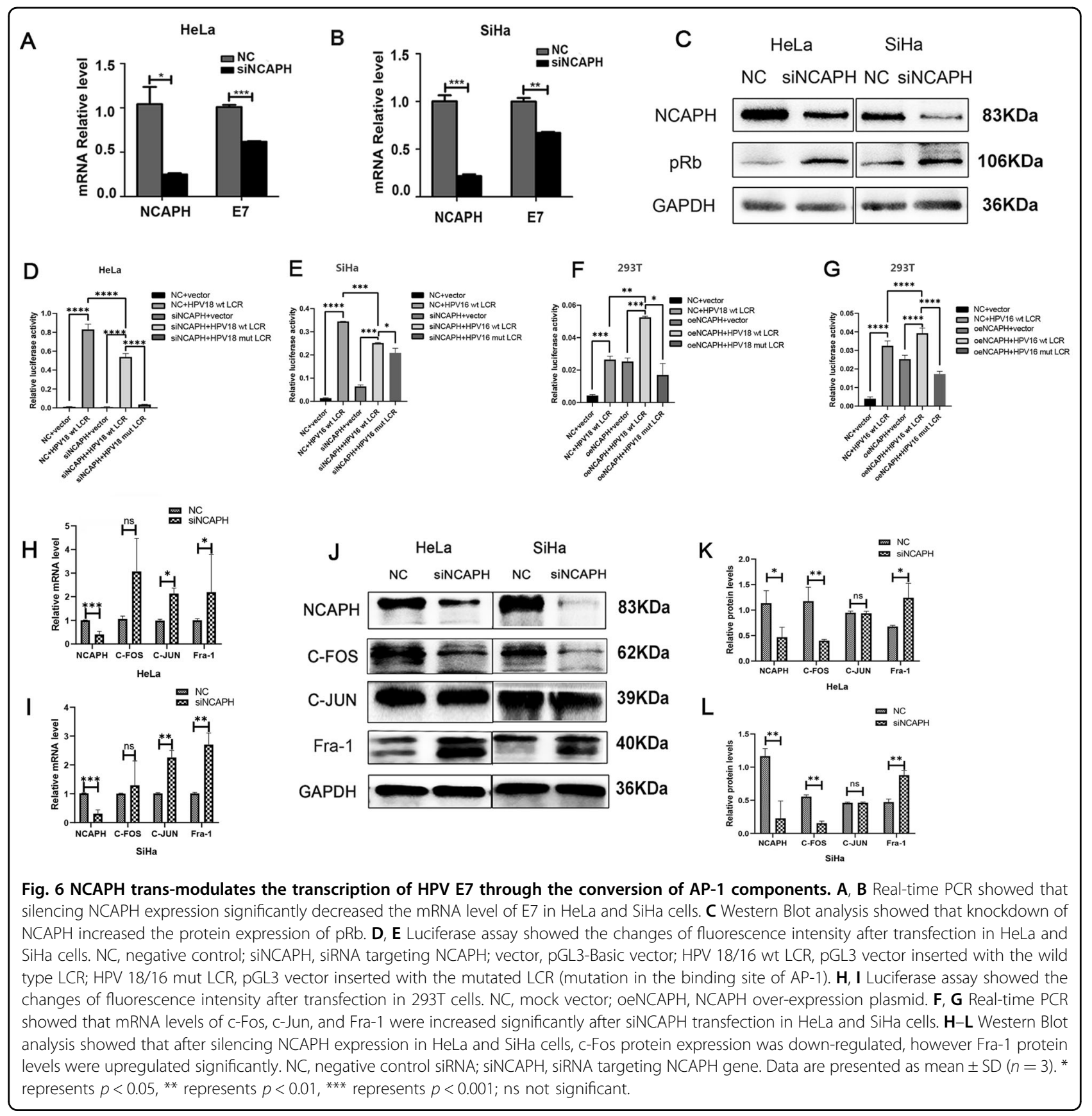

HPV E7 were reduced (Fig. 6A, B) and pRb protein level increased significantly (Fig. 6C-E). On the basis of our finding that $\mathrm{E} 7$ regulates the transcription of $\mathrm{NCAPH}$, this result suggests that a positive feedback loop exists between HPV E7 and NCAPH.

Previous studies have shown that the LCR region of HPV E7 often acts as the binding sites for transcription factors and plays an essential role in the regulation of HPV gene expression. To elucidate whether NCAPH participates in the transcription regulation of E7 expression through the LCR region, luciferase assay was performed in HeLa, SiHa and 293T cells. Results showed that the fluorescence intensity increased greatly after the transfection of HPV 18 or 16 full-length LCR (Fig. 6D-G). It suggested that the viral promoter was active in all three kinds of cells, and HPV integration into host genome did not completely destroy the activity of HPV LCR in HeLa and SiHa. Moreover, when we knocked-down NCAPH in $\mathrm{HeLa}$ and $\mathrm{SiHa}$ transfected with the LCR, the fluorescence signal reduced significantly, and when we over-expressed 
NCAPH in 293T transfected with the LCR, the signal was further enhanced (Fig. 6D-G). The effects of NCAPH on the fluorescence intensity indicated that NCAPH was able to activate the transcription of the LCR. NCAPH might regulate $\mathrm{E} 7$ gene transcription by enhancing the activity of viral promoter in cervical cancer cells.

AP-1 is a key transcription factor that regulates E7 transcription by binding to LCR, and the conversion of AP-1 heterodimer regulates LCR activity significantly. Consistent with it, when we mutated the binding sites of AP-1 in HPV 16 and 18 LCR, the fluorescence intensity decreased greatly compared with the control group (Fig. 6 D-J). To unravel whether AP-1 participates in NCAPHmediated LCR activation, we investigated the component changes of AP-1 transcription factor after interference with NCAPH in cervical cancer cells. Results showed that after interfering the expression of NCAPH, mRNA levels of c-Jun, c-Fos and Fra-1 were increased in HeLa and $\mathrm{SiHa}$ cells (Fig. 6H, I). At protein level, after silencing NCAPH expression in HeLa cells, c-Fos was down-regulated, while Fra-1 was up-regulated significantly; however, no significant change was observed for c-Jun level (Fig. 6J, K). Similar results were obtained in SiHa cells (Fig. 6J, L). These data indicated that silencing NCAPH could induce the conversion of AP-1 heterodimer from c-Fos/c-Jun to Fra-1/c-Jun, thus reduce its binding activity to HPV LCR and down-regulates HPV E7 transcription.

\section{Potential NCAPH involvement in the activation of the PI3K/ AKT/SGK signaling pathway}

Analysis of UALCAN data showed that PDK1 and SGK3 were over-expressed in cervical cancer compared with normal cervix (Figs. 7A and 8). Analysis of GEO data (GSE7803) showed that the expression of PDK1 in cervical cancer was significantly higher than in normal cervix and HSIL separately (Fig. 7B). The data from cBioportal database revealed that $\mathrm{NCAPH}$ was significantly coexpressed with PDK1 in cervical cancer specimens (Fig. 7C).

To verify the above results, we examined whether NCAPH was involved in the regulation of the PI3K/AKT/ SGK signaling pathway. Results showed that when the NCAPH expression was knocked down in HeLa and SiHa cells, the levels of AKT, SGK3, P70S6K and mTOR were unchanged, however the levels of PDK1, p-AKT(Ser473), p-SGK3(320), p-P $\mathrm{P}^{70 S 6 \mathrm{~K}}$ and p-mTOR were significantly decreased (Fig. 7D-F) (all $p$ values $<0.05$ ). These changes in the level of key signaling molecules suggest that NCAPH might play a key role in the activation of the PI3K/AKT/SGK signaling pathway.

Furthermore, we compared RPE1-pbabe cells with RPE1-16E7 cells. The results showed that the increase of $\mathrm{NCAPH}$ in E7 cells was accompanied by the increase of $\mathrm{p}-$ AKT(Ser473) protein level, however, the total Akt level was not changed. When we knocked down $\mathrm{NCAPH}$ expression in RPE1-16E7 cells, the protein level of p-AKT (Ser473) decreased significantly (Fig. 7G-I). These results suggest that E7 may be involved in activation of PI3K/ AKT/SGK signaling pathway by activating Akt phosphorylation at ser473 site through NCAPH.

\section{Discussion}

NCAPH is considered to be a protein involved in sister chromatid isolation during mitosis. At present, little is known about its role in human tumors. In this study, we have shown that silencing NCAPH expression reduced the proliferation, invasion, migration, and xenograft tumor formation ability of cervical cancer cells in vitro and in vivo. These factors imply that NCAPH could have an important role in promoting the progression of cervical cancer. These results are consistent with previous observations reported for colorectal cancer cells, lung cancer cells and breast cancer cells ${ }^{18,19,24}$. Therefore, NCAPH might be an essential oncogene not limited to cervical cancer more widely involved in the development and progression of various malignancies.

HPV E7 protein is a key virus-encoded carcinogenic factor. E7 can degrade $\mathrm{pRb}$ and release E2F, thereby regulating growth cycle and DNA repair, and inducing genomic instability. It has been proven that pRB, E2F1, and condensin II can localize to major satellite repeats at pericentromeres, forming a complex that ensures proper chromosome segregation in mitosis. These results suggest a possible association amongst E7, E2F1, and condensing proteins ${ }^{25}$. We found that E7 could enhance NCAPH gene expression by promoting the direct binding of E2F1 to the promoter of NCAPH gene. Considering the important role of NCAPH in the condensin complex, we speculate that although E7 up-regulated E2F1 and $\mathrm{NCAPH}$ expression, the degradation of $\mathrm{pRb}$ by E7 hinders the formation of pRb-E2F1-condensin complex, which leads to incorrect chromosome segregation, genomic instability, and tumorigenesis. However, the hypothesis needs to be further verified.

In recent years, researchers have made great progress in understanding the functions of E6 and E7. However, past researches mainly focused on how E7 regulates the expression of host genes, but how host genes transregulate the expression of E7 is still largely unknown ${ }^{26}$. Moreover, in order to resist the transformation of cells by the virus, most host proteins are proved to act as limiting factors to inhibit the replication and expression of HPV genes $^{26}$. Different from previous studies, the mRNA level of E7 decreased significantly when we knocked down $\mathrm{NCAPH}$ in cervical cancer cells. It indicates that NCAPH can trans-modulate the transcription of E7. Considering the positive regulatory effect of $\mathrm{E} 7$ on $\mathrm{NCAPH}$, this result suggests that a feedback loop exists between HPV E7 and NCAPH. However, the E7 protein is often considered to 


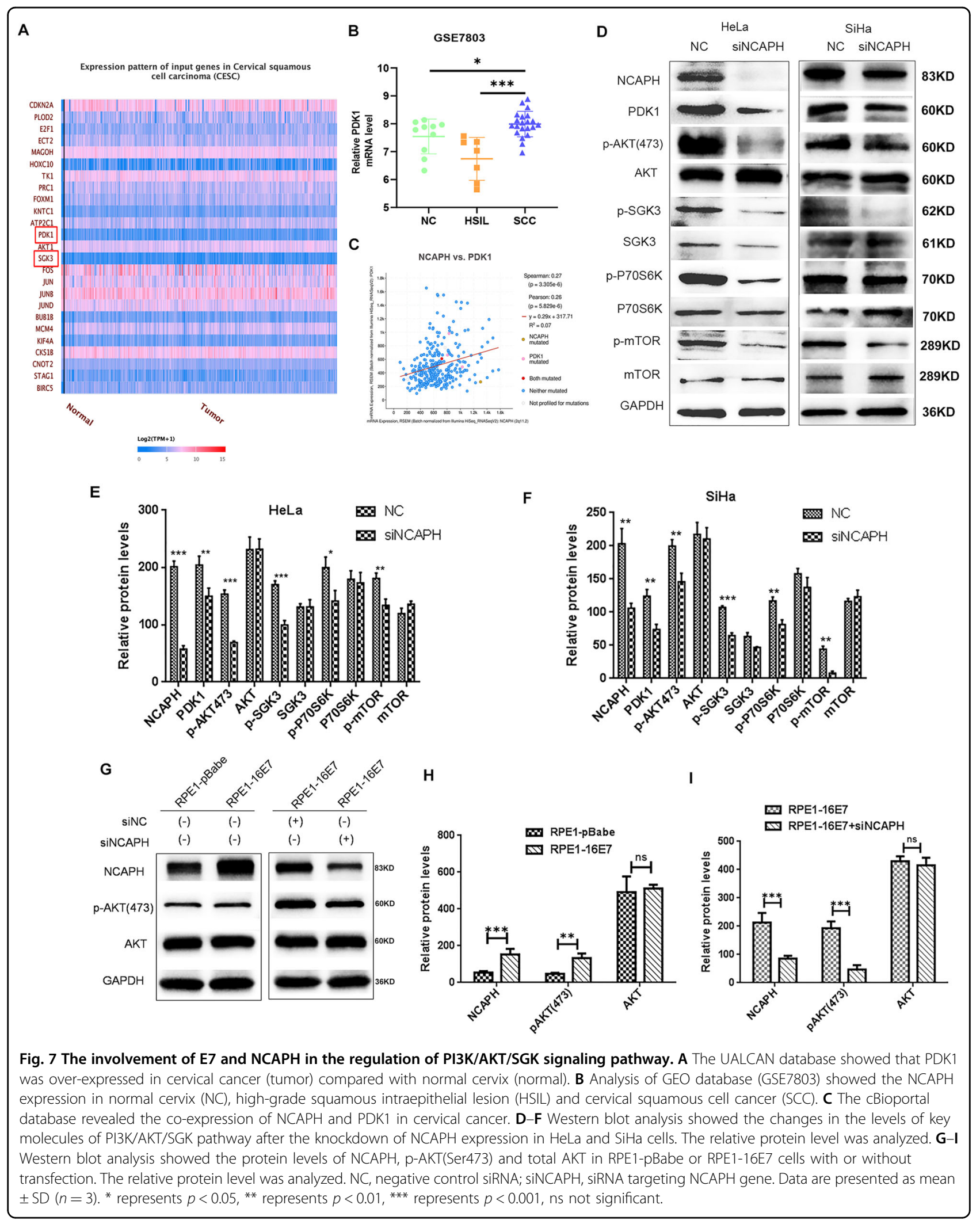




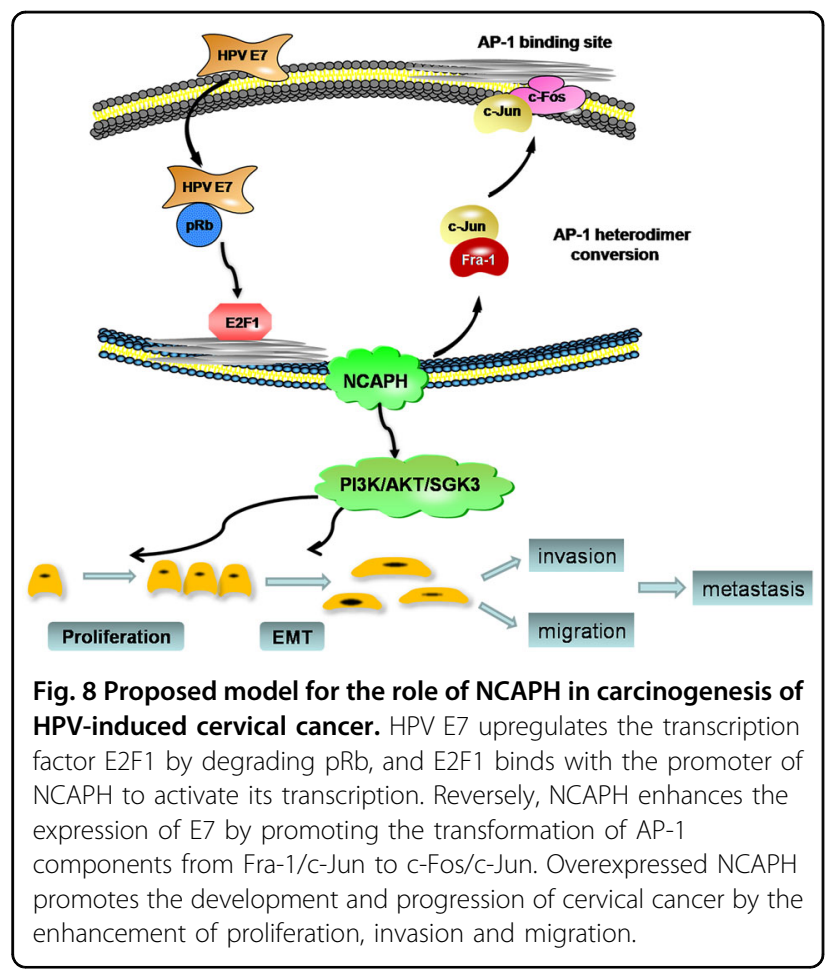

be a double-edged sword: the expression of E7 increases cell proliferation, while its overexpression induces cell death $^{3}$. Similarly, the overexpression of NCAPH in cervical cancer cells induced severe cell death (data not shown). This suggests that the positive feedback loop of E7-NCAPH in cervical cancer cells is in equilibrium under the control of host cells. The overexpression of $\mathrm{NCAPH}$ might break the balance and upregulate the expression of E7, which eventually leads to cell death.

It is known that HPV genome is integrated into host chromosome in cervical cancer cells, however it is unclear whether NCAPH regulates E7 through host or viral promoters. Our data confirmed that the viral promoter was still active in cervical cancer cells which was consistent with previous reports ${ }^{27}$. Moreover, NCAPH could upregulate the activity of HPV LCR significantly. It indicated that NCAPH could regulate E7 through viral promoter. AP-1 is a key transcription factor responsible for the regulation of E7 promoter activity. It forms a heterodimer composed of Fos gene family (c-Fos, FosB, Fra-1, Fra-2) and Jun gene family (c-Jun, JunB, or JunD) ${ }^{28,29}$. During the malignant transformation of cervical cells, AP-1 complex transforms from Fra-1/c-Jun to c-Fos/c-Jun dimer, enhances the transcription of $\mathrm{E} 7$, and promotes the carcinogenesis process ${ }^{30-32}$. Consistent with it, we found that the mutation of AP-1 binding site in HPV LCR reduced the activity of LCR significantly. We speculated that NCAPH might regulate E7 transcription through AP-1. In line with this, we found that interference with $\mathrm{NCAPH}$ can significantly reduce the expression of c-Fos and increase the expression of Fra-1. It indicates that silencing NCAPH could induce the conversion of AP-1 heterodimers from c-Fos/c-Jun to Fra-1/c-Jun, reduce the activity of AP-1, and inhibit the transcription of E7. Moreover, the changes of AP-1 mRNA level after NCAPH interference were not completely consistent with the changes of protein level. It suggests that the regulation of $\mathrm{NCAPH}$ on c-Fos, c-Jun, and Fra-1gene expression may be complex, and its mechanism needs to be further clarified.

The PI3K/AKT pathway is an important signaling pathway, and there is clear evidence that amplification or mutation of the main components of PI3K/AKT pathway is common in cervical cancer and contributes to tumor progression $^{9,33-38}$. HPV E6 and E7 could change intracellular and molecular events through PI3K/AKT signaling pathway, and promote malignant transformation of cervical cells ${ }^{37}$. However, the specific mechanisms are still unclear. In our study, we found that E7 was involved in the activation of PI3K/AKT signaling pathway partially through NCAPH. Silencing of NCAPH did not change the levels of AKT, SGK3, P70S6K, and mTOR, but significantly decreased their phosphorylation levels. The reduction in PDK1 expression when NCAPH expression is reduced suggests that, NCAPH could potentially activate the phosphorylation of AKT and SGK3 via upregulation of PDK1 expression. Bago et al. reported that AKT inhibitors increased the activation of SGK3 in the treatment of breast cancer, and SGK3 replaces AKT by phosphorylated TSC2, leading to the failure of AKT inhibitor treatment in some breast cancer patients ${ }^{39}$. Therefore, future therapy targeting NCAPH might have the potential to inhibit AKT and SGK simultaneously and exhibit better effects than AKT or SGK inhibitor treatment alone.

In cervical cancer, EMT plays an essential role in initiating cancer progression ${ }^{40}$. In the present study, silencing of NCAPH hindered the EMT process. The tight correlation between NCAPH and EMT was further confirmed in the xenograft tumor model suggesting NCAPH may promote EMT in cervical cancer cells. Furthermore, it is known that cells with a mesenchymal phenotype are prone to invading the lymphvascular cavities and enabling metastasis to distant sites ${ }^{41}$. The expression of NCAPH has consistently been associated with lymph node metastasis in our cervical cancer patients indicating that NCAPH might facilitate tumor invasion and metastasis by promoting EMT.

Recently, Zhai $\mathrm{Y}$ et al. revealed that NCAPH mRNA increased significantly from normal cervix to HSIL and from HSIL to ICSCC using high-density oligonucleotide microarrays. However, the results required further functional validation ${ }^{42}$. In our study, we assessed the 
expression of NCAPH in human cervical cancer tissues. We found NCAPH protein to be located both in cytoplasm and nucleus, a result that was consistent with a previous study that condensin I was present in the cytoplasm during interphase, and gained access to chromosomes only after breakdown of the nuclear envelope at the end of prophase ${ }^{43}$. Moreover, NCAPH expression in cervical cancer was significantly higher than those in normal cervix and HSIL. This result was in accordance with the observation in colon cancer patients ${ }^{18}$ and provides further evidence that NCAPH plays an important role in the initiation of cervical cancer. Although positive $\mathrm{NCAPH}$ expression was significantly associated with lymph node metastasis, its expression correlated with a better patient prognosis. This was opposite to the effect observed for non-small cell lung cancer ${ }^{16}$ while consistent with that in colon cancer ${ }^{18}$. Analysis of our patient cohort showed that the majority of the patients received adjuvant therapy because of high risk factors, such as lymph node metastasis (34/100) and deep invasion (74/100). Considering the vital role of NCAPH in cell proliferation, we hypothesized that tumors with positive NCAPH expression might have a higher proliferation rate, which could improve the effects of chemo- and/or radio-therapies and bring benefits to the patients' prognosis. However, the result needs to be re-assessed in larger cohort of patients in the future.

In summary, we report that NCAPH is a novel oncogene that plays an important role in the initiation and progression of cervical cancer. The formation mechanism of feedback loop between HPV E7 and NCAPH deepens the molecular mechanism of HPV induced cervical cancer, and expands the mechanism of trans-regulation of host protein to HPV genes. In addition, NCAPH might be a novel multi-functional therapeutic target. Future personalized therapies targeting $\mathrm{NCAPH}$ is expected to induce robust antitumor responses not only reducing tumor growth ability, but also decreasing the expression of E7.

\section{Acknowledgements}

This study was supported by Shandong Natural Science Foundation (grant no. ZR2016HM18), Postdoctoral Funds in China (2014M560560), China-Australia Centre for Health Sciences Research (CACHSR)(2015GJ03 and 2018GJ06).

\footnotetext{
Author details

${ }^{1}$ Department of Pathology, School of Basic Medical Sciences, Shandong University, Jinan, PR China. '2Department of Radiation Oncology, Qilu Hospital, Cheeloo College of Medicine, Shandong University, Jinan, PR China. ${ }^{3}$ Department of Pathology, Qilu Hospital, Cheeloo College of Medicine, Shandong University, Jinan, PR China. ${ }^{4}$ Experimental Therapeutics Laboratory, Clinical and Health Sciences, University of South Australia Cancer Research Institute, Adelaide, SA, Australia. ${ }^{5}$ Key Laboratory for Experimental Teratology of Chinese Ministry of Education, The Shandong Provincial Key Laboratory of Infection and Immunology, Department of Microbiology, School of Basic Medical Sciences, Shandong University, Jinan, PR China
}

\section{Author contributions}

X.W. conceived the experiments. M.W., X.W.Q., W.P., J.X.L., and X.J.C. carried out the experiments. Y.B.Z., S.L.Z., Y.Z., and R.R.Z. analyzed the data. T.C., L.L., and J.H. were involved in writing the paper. All the authors had final approval of the submitted and published versions.

\section{Conflict of interest}

The authors declare that they have no conflict of interest.

\section{Publisher's note}

Springer Nature remains neutral with regard to jurisdictional claims in published maps and institutional affiliations.

Supplementary Information accompanies this paper at (https://doi.org/ 10.1038/s41419-020-03244-9).

Received: 19 May 2020 Revised: 9 November 2020 Accepted: 11 November 2020

Published online: 11 December 2020

\section{References}

1. Ojesina, A. I. et al. Landscape of genomic alterations in cervical carcinomas, Nature 506, 371-375 (2014).

2. Bodily, J. \& Laimins, L. A. Persistence of human papillomavirus infection: keys to malignant progression. Trends Microbiol. 19, 33-39 (2011).

3. Roman, A. \& Munger, K. The papillomavirus E7 proteins. Virology 445, 138-168 (2013).

4. Hanning, J. E. et al. Depletion of HPV16 early genes induces autophagy and senescence in a cervical carcinogenesis model, regardless of viral physical state. J. Pathol. 231, 354-366 (2013).

5. Park, J. S. et al. Inactivation of interferon regulatory factor-1 tumor suppressor protein by HPV E7 oncoprotein: implication for the E7-mediated immune evasion mechanism in cervical carcinogenesis. J. Biol. Chem. 275, 6764-6769 (2000).

6. Zeng, Q. et al. LKB1 inhibits HPV-associated cancer progression by targeting cellular metabolism. Oncogene 36, 1245-1255 (2017).

7. Matarrese, P. et al. Interaction between the human papillomavirus 16 E7 oncoprotein and gelsolin ignites cancer cell motility and invasiveness. Oncotarget 7, 50972-50985 (2016).

8. Narisawa-Saito, M. \& Kiyono, T. Basic mechanisms of high-risk human papillomavirus-induced carcinogenesis: roles of E6 and E7 proteins. Cancer Sci. 98, 1505-1511 (2007). Epub 2007 Jul 23.

9. Charette, S. T. \& McCance, D. J. The E7 protein from human papillomavirus type 16 enhances keratinocyte migration in an Akt-dependent manner. Oncogene 26, 7386-7390 (2007). Epub 2007 May 28.

10. Halim, T. A., Farooqi, A. A. \& Zaman, F. Nip the HPV encoded evil in the cancer bud: HPV reshapes TRAlls and signaling landscapes. Cancer Cell Int. 13, 61 (2013).

11. Schwarz, J. K. et al. Pathway-specific analysis of gene expression data identifies the PI3K/Akt pathway as a novel therapeutic target in cervical cancer. Clin. Cancer Res. 18, 1464-1471 (2012).

12. Hirano, T. HEAT repeats associated with condensins, cohesins, and other complexes involved in chromosome-related functions. Genome Res. 10 1445-1452 (2000).

13. Hirano, T. Condensins: universal organizers of chromosomes with diverse functions. Genes Dev. 26, 1659-1678 (2012).

14. Wood, A. J., Severson, A. F. \& Meyer, B. J. Condensin and cohesin complexity: the expanding repertoire of functions. Nat. Rev. Genet. 11, 391-404 (2010).

15. Ryu, B. et al. Comprehensive expression profiling of tumor cell lines identifies molecular signatures of melanoma progression. PLOS ONE 2, e594 (2007).

16. Perumal, D., Singh, S., Yoder, S. J., Bloom, G. C. \& Chellappan, S. P. A novel five gene signature derived from stem-like side population cells predicts overall and recurrence-free survival in NSCLC. PLOS ONE 7, e43589 (2012).

17. Cui, F., Hu, J., Xu, Z., Tan, J. \& Tang, H. Overexpression of NCAPH is upregulated and predicts a poor prognosis in prostate cancer. Oncol. Lett. 17, 5768-5776 (2019).

18. Yin, L. et al. NCAPH plays important roles in human colon cancer. Cell Death Dis. 8, e2680 (2017). 
19. Li, Q. G. et al. A normalization-free and nonparametric method sharpens largescale transcriptome analysis and reveals common gene alteration patterns in cancers. Theranostics 7, 2888-2899 (2017).

20. Sun, C. et al. Non-SMC condensin I complex subunit H enhances proliferation, migration, and invasion of hepatocellular carcinoma. Mol. Carcinog. 58, 2266-2275 (2019).

21. Kim, J. H., Youn, Y., Kim, K. T., Jang, G. \& Hwang, J. H. Non-SMC condensin I complex subunit $\mathrm{H}$ mediates mature chromosome condensation and DNA damage in pancreatic cancer cells. Sci. Rep. 9, 17889 (2019).

22. Juan, L. et al. Cancerous inhibitor of protein phosphatase $2 \mathrm{~A}$ is overexpressed in cervical cancer and upregulated by human papillomavirus 16 E7 oncoprotein. Gynecol. Oncol. 122, 430-436 (2011).

23. Chen, $Y$. et al. INPP4B restrains cell proliferation and metastasis via regulation of the PI3K/AKT/SGK pathway. J. Cell Mol. Med. 22, 2935-2943 (2018).

24. Xiong, Y. C., Wang, J., Cheng, Y., Zhang, X. Y. \& Ye, X. Q. Overexpression of MYBL2 promotes proliferation and migration of non-small-cell lung cancer via upregulating NCAPH. Mol. Cell Biochem. 468, 185-193 (2020).

25. Coschi, C. H. et al. Haploinsufficiency of an RB-E2F1-Condensin II complex leads to aberrant replication and aneuploidy. Cancer Discov. 4, 840-853 (2014).

26. Porter, S. S., Stepp, W. H., Stamos, J. D. \& McBride, A. A. Host cell restriction factors that limit transcription and replication of human papillomavirus. Virus Res. 231, 10-20 (2017).

27. Chow, W. Y. et al. A novel MLL5 isoform that is essential to activate E6 and E7 transcription in HPV16/18-associated cervical cancers. Cancer Res. 71, 6696-6707 (2011).

28. Bodily, J. M., Mehta, K. P., Cruz, L., Meyers, C. \& Laimins, L. A. The E7 open reading frame acts in cis and in trans to mediate differentiation-dependent activities in the human papillomavirus type 16 life cycle. J. Virol. 85, 8852-8862 (2011).

29. Eferl, R. \& Wagner, E. F. AP-1: a double-edged sword in tumorigenesis. Nat. Rev. Cancer 3, 859-868 (2003).

30. Wilde, J. et al. Alterations in AP-1 and AP-1 regulatory genes during HPVinduced carcinogenesis. Cell Oncol. 30, 77-87 (2008).
31. Butz, K. \& Hoppe-Seyler, F. Transcriptional control of human papillomavirus (HPV) oncogene expression: composition of the HPV type 18 upstream regulatory region. J. Virol. 67, 6476-6486 (1993).

32. Rösl, F., Das, B. C., Lengert, M., Geletneky, K. \& zurHausen, H. Antioxidantinduced changes of the AP-1 transcription complex are paralleled by a selective suppression of human papillomavirus transcription. J. Virol. $\mathbf{7 1}$ 362-370 (1997).

33. Toker, A. mTOR and Akt signaling in cancer: SGK cycles in. Mol. Cell. 31, 6-8 (2008).

34. Lien, E. C., Dibble, C. C. \& Toker, A. PI3K signaling in cancer: beyond AKT. Curr. Opin. Cell Biol. 45, 62-71 (2017).

35. Vasudevan, K. M. et al. AKT-independent signaling downstream of oncogenic PIK3CA mutations in human cancer. Cancer Cell. 16, 21-32 (2009).

36. Feng, $\mathrm{T}$. et al. Growth factor progranulin promotes tumorigenesis of cervical cancer via PI3K/Akt/mTOR signaling pathway. Oncotarget 7, 58381-58395 (2016).

37. Wu, J. et al. Four major factors regulate phosphatidylinositol 3-kinasesignaling pathway in cancers induced by infection of human Papillomaviruses. Curr. Med. Chem. 21, 3057-3069 (2014).

38. Zhang, L. et al. The role of the PI3K/Akt/mTOR signaling pathway in human cancers induced by infection with human papillomaviruses. Mol. Cancer 14, 87 (2015).

39. Bago, R. et al. The hVps34-SGK3 pathway alleviates sustained PI3K/Akt inhibition by stimulating mTORC1 and tumour growth. EMBO J. 35, 1902-1922 (2016).

40. Qureshi, R., Arora, H. \& Rizvi, M. A. EMT in cervical cancer: its role in tumour progression and response to therapy. Cancer Lett. 356, 321-331 (2015).

41. May, M. et al. A switch from epithelial to mesenchymal properties correlates with lymphovascular invasion in squamous cell carcinoma of the penis. Pathol Res Pract 211, 641-645 (2015).

42. Zhai, Y. et al. Gene expression analysis of preinvasive and invasive cervical squamous cell carcinomas identifies HOXC10 as a key mediator of invasion. Cancer Res. 67, 10163-10172 (2007).

43. Ono, T. et al. Differential contributions of condensin I and condensin II to mitotic chromosome architecture in vertebrate cells. Cell 115, 109-121 (2003). 